\title{
SETTLING A NUMBER OF QUESTIONS ABOUT HYPER-ARCHIMEDEAN LATTICE-ORDERED GROUPS
}

\author{
PAUL CONRAD AND JORGE MARTINEZ
}

(Communicated by Louis J. Ratliff, Jr.)

\begin{abstract}
This note contains the answers to several long-standing questions about hyper-Archimedean lattice-ordered groups. In particular, we construct such a lattice-ordered group which admits no embedding into an Archimedean lattice-ordered group with a strong order unit.
\end{abstract}

\section{INTRODUCTION}

A lattice-ordered group $(G,+, \vee, \wedge)$-abbreviated, as is the custom in the literature: $\ell$-group-is a group with an underlying lattice order, so that $a+$ $(b \vee c)=(a+b) \vee(a+c)$ holds for all $a, b$, and $c$ in $G$, as well as its latticetheoretic dual. The meaning of the terms $\ell$-subgroup, $\ell$-homomorphism, and $\ell$-isomorphism should be obvious to the reader.

The cardinal product, $\prod\left\{G_{i}: i \in I\right\}$ of the $\ell$-groups $G_{i}$, is the Cartesian product of the $G_{i}$ with pointwise operations. In this context, the cardinal sum, denoted $\sum\left\{G_{i}: i \in I\right\}$ or else $\boxplus\left\{G_{i}: i \in I\right\}$ if formed internally, is the $\ell$ subgroup of the cardinal product consisting of the finitely nonzero functions.

An $\ell$-group $G$ is Archimedean if for each pair $a, b \geq 0$ there is a natural number $n$ so that $n a \not b$. It is well known that every Archimedean $\ell$-group is Abelian. (For this and other basic results from the theory of $\ell$-groups we refer the reader to [AF] or [BKW].) An $\ell$-group is hyper-Archimedean if each $\ell$ homomorphic image is Archimedean. The class of hyper-Archimedean $\ell$-groups is reasonably well understood. $\left[\mathrm{C}_{3}\right]$ gives a thorough account of these $\ell$-groups; we shall refer to it as needed in the sequel. For instance, it is known that an $\ell$-group $G$ is hyper-Archimedean if and only if $G$ has a representation as an $\ell$ subgroup of a cardinal product of subgroups of the reals, say $G \subseteq \prod\left\{R_{i}: i \in I\right\}$, identifying $G$ with its image in the product, so that for each pair of functions $0<f, g \in G$ there is an $n \in \mathbf{N}$ for which $n f_{i}>g_{i}$ for all $i \in I$ on which $f_{i}>0$. Moreover, each representation of $G$ by real-valued functions has this property.

Received by the editors February 21, 1989 and, in revised form, May 23, 1989.

1980 Mathematics Subject Classification (1985 Revision). Primary 06F20; Secondary 04A25.

(C) 1990 American Mathematical Society $0002-9939 / 90 \$ 1.00+\$ .25$ per page 
In the theory of $\ell$-groups one distinguishes between strong units and (ordinary) units. $0<u \in G$ is a unit if $u \wedge g>0$ for each $g>0$. We call $u$ a strong unit if for each $g>0$ there is a natural number $n$ so that $n u \geq g$; (or, equivalently, if the convex $\ell$-subgroup $G(u)$ generated by $u$ is $G$. The role played by the lattice of convex $\ell$-subgroups in the theory of $\ell$-groups is a very important one; the reader should refer to either [AF] or [BKW].) Clearly, every strong unit is a unit, and from the characterization of hyper-Archimedean $\ell$-groups mentioned in the preceding paragraph, it should be clear that if $G$ is hyper-Archimedean then every unit of $G$ is a strong unit. Note as well that if $G$ is hyper-Archimedean with unit $u>0$, then the representation discussed above can be arranged so that $u$ is represented by the constant function 1 . Once this has been done it should be evident that if $g>0$ then, viewed as a function, $\left\{g_{i}: g_{i}>0\right\}$ is bounded above and has a strictly positive lower bound. (Putting it differently: the positive range of $g$ is contained in an interval $(a, b)$ with $a>0$.)

Conrad asked in $\left[\mathrm{C}_{3}\right]$-open question (2)-if this kind of representation could be obtained for every hyper-Archimedean $\ell$-group, (regardless of whether or not there was as unit in the group.) The example we will present shows that the answer to this question is no. In remarks following the construction of this example we will indicate how it also answers other questions from $\left[\mathrm{C}_{3}\right]$ in the negative.

\section{AlMOST DISJOINT SETS}

We consider families of infinite subsets of the set $\mathbf{N}$ of natural numbers; suppose $\mathbf{M}$ is such a family with the feature that if $A, B \in \mathbf{M}$ and $A \neq B$, then $A \cap B$ is finite. We shall call such sets almost disjoint. For each $A \in \mathbf{M}$ let $v_{A}$ be an integer-valued sequence so that $v_{A}(n)>0$ precisely when $n \in A$, and 0 otherwise. Let $G=G(\mathbf{M})$ be the subgroup of $\Pi\{Z: n \in \mathbf{N}\}$ generated by $\left\{v_{A}: A \in \mathbf{M}\right\}$ and $\sum\{Z: n \in \mathbf{N}\}$. The following claims are either obvious or else straightforward to verify:

A. $G$ is a free Abelian group on the generators $\left\{v_{A}: A \in \mathbf{M}\right\}$ together with the $v_{n}$, the characteristic functions of each singleton $\{n\}$.

B. $G$ is an $\ell$-subgroup of $\prod\{Z: n \in \mathbf{N}\}$.

C. $G$ is hyper-Archimedean. (A fact guaranteed by the almost-disjointness.)

Recall that an $\ell$-group is Specker if it is generated as a group by its singular elements; a positive element $s$ is singular if $x \wedge(s-x)=0$, for each $0 \leq x \leq s$. In a subdirect product of copies of the group of integers $Z$, the singular elements are precisely the characteristic functions. A Specker group has a natural ring structure: there is, in fact, a unique multiplication so that $s t=s \wedge t$, for all singular elements; see $\left[\mathrm{C}_{3}\right]$. Thus when viewed as a ring the singular elements are precisely the idempotents.

D. $G$ is a Specker group if and only if for each $A \in \mathbf{M}, v_{A}(n)=1$ for all but a finite number of $n \in A$. (We shall say that $v_{A}$ is almost characteristic.) 
The $v$-hull $A^{\prime \prime}$ of an Archimedean $\ell$-group $A$ is the unique minimal vector lattice in which $A$ is large; that is, so that each nonzero convex $\ell$-subgroup of $A^{v}$ intersects $A$ nontrivially. (We refer the reader to $\left[\mathrm{B}_{1}\right]$ or $\left[\mathrm{C}_{2}\right]$, and also to $\left[\mathrm{C}_{3}\right]$.)

E. The $v$-hull $G^{v}$ of $G$ is the subspace of real sequences spanned by the $v_{A}(A \in \mathbf{M})$ and the $v_{n}(n \in \mathbf{N})$; these then form a basis for $G^{v}$.

F. $G$ is an extension of one cardinal sum of copies of the integers by another. (Simply factor out the subgroups of finitely nonzero sequences.)

An element $b>0$ of an $\ell$-group $L$ is said to be basic if the convex $\ell$ subgroup $L(b)$ generated by $b$ is totally ordered; this is equivalent to requiring that the set $\{x \in L: 0 \leq x \leq b\}$ be a chain. (The reader is referred to [AF] or [BKW] for additional material on basic elements.) For now we need to remind the reader that $\left\{b_{i}: i \in I\right\}$ is called a basis if this set is maximal with respect to the property that its elements are pairwise disjoint, and if each $b_{i}$ is basic.

The following lemma is crucial in the subsequent development:

Lemma. Suppose that $A$ is an Archimedean $\ell$-group with a basis $\left\{a_{i}: i \in I\right\}$. Then $A$ can be represented as an $\ell$-subgroup of the cardinal product $P=$ $\Pi\{R: i \in I\}$ of copies of the reals so that $A$ contains all the finitely nonzero functions $f$ for which $f(i) \in A\left(a_{i}\right)$. Moreover, if $A$ is contained in an Archimedean $\ell$-group $B$ possessing a strong unit, then there is an $\ell$-subgroup $C$ of $P$ containing a strong unit and $A$.

(Note: Since the first assertion is nothing new, let us emphasize the significance of this result: the point is that if it is possible to adjoin a strong unit to $A$, and $A$ has a basis, then it is (essentially) possible to adjoin a strong unit in any given representation arising from a basis.)

Proof. As we just said, it is well known that the representation claimed in the first statement can be achieved: the reason is that for each basic element $a \in A$, $A=A(a) \boxplus a \perp$, where $a \perp$ stands for the polar of $a$. (The reader might look at Theorem 7.3 in $\left[\mathrm{C}_{1}\right]$ or else Theorem 11.1 .12 in [BKW].) We obviously identify $A$ with its image in $P$.

Since $B$ has a strong unit, the intersection of all maximal convex $\ell$-subgroups of $B$ is trivial, and therefore $B$ can be embedded as an $\ell$-subgroup of a cardinal product $Q=\prod\{\mathbf{R}: k \in K\}$. Let us call this embedding map $\sigma$; evidently, $\sigma A \subseteq \sigma B \subseteq Q$.

For each $a_{i}$ pick an element $k(i)$ in $K$ in the support of $\sigma\left(a_{i}\right)$. Let $K^{*}=$ $\{k(i): i \in I\}$ and $Q^{*}=\prod\{\mathbf{R}: k \in K\}$. Clearly, the map $\sigma^{*}$, obtained by following $\sigma$ with the projection of $Q$ upon $Q^{*}$, is one-to-one. We now have $\sigma^{*} A \subseteq \sigma^{*} B \subseteq Q^{*}$. Thus, without loss of generality, we may assume that $K=K^{*}$. (We can and will, therefore, drop all the ${ }^{*}$, since the distinction is meaningless after $K$ has been identified with $K^{*}$.)

We also identify $I$ with $K$, since the map $i \rightarrow k(i)$ is one-to-one. The latter extends to an $\ell$-automorphism $\tau$ of $P$. Now, in the language of the ConradHarvey-Holland embedding theorem-see $[\mathrm{CHH}],[\mathrm{AF}]$, or else $[\mathrm{BKW}]-$ the 
map $\tau^{-1} \sigma$ is a value-preserving embedding of $A$ into $P$, and hence, by that theorem, $\tau^{-1} \sigma$ is the restriction of an $\ell$-automorphism $\alpha$ of $P$.

Now set $C=\alpha^{-1} \tau^{-1} \sigma B$, and we are finished.

We shall construct an example of a hyper-Archimedean $\ell$-group which cannot be embedded in a hyper-Archimedean $\ell$-group with unit, by applying the preceding lemma to $G(\mathbf{M})$, as constructed prior to the lemma, for a very particular kind of almost disjoint family $\mathbf{M}$.

Suppose we consider the binary tree:

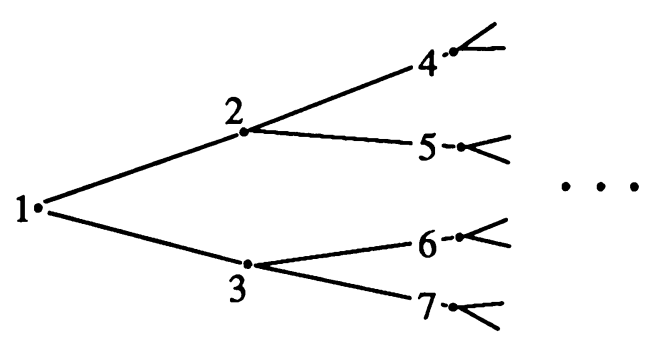

and the almost disjoint family $\mathbf{M}$ of infinite subsets of $\mathbf{N}$ determined by the maximal chains-or branches-through the tree. The reader should find it convenient, as we do, to think, in the sequel, of the sets in $\mathbf{M}$ as increasing sequences of natural numbers. Observe then that in the coordinatewise ordering of such sequences all members of $\mathbf{M}$ are bounded by the sequence $\left(1,3,7, \ldots, 2^{n}-1, \ldots\right)$. For our application of the lemma to work it is this aspect of boundedness that seems to be important. Note also that $\mathbf{M}$ has the cardinality of the continuum.

So let us assume from now on that $\mathbf{M}$ is any family of almost disjoint increasing sequences of natural numbers which we shall label $s(\delta)=(s(1, \delta)$, $s(2, \delta), \ldots)$, and where $\delta$ ranges over an index set with the cardinality of the continuum. We assume that there is a sequence $t=(t(1), t(2), \ldots)$ so that for each $\delta$ and each $n \in \mathbf{N}, s(n, \delta) \leq t(n)$.

Next, use the same indexing to denote the family of all increasing sequences of natural numbers. Putting it differently: we fix a one-to-one correspondence $s(\delta) \rightarrow f(\delta)$ between the members of $\mathbf{M}$ and the family of all increasing sequences of natural numbers. (As the reader will soon appreciate, we do want all the increasing sequences.)

Now define, for each $\delta$, a positive element in $P=\prod\{\mathbf{Z}: n \in \mathbf{N}\}$ as follows:

$$
v(k, \delta)= \begin{cases}0, & \text { if } k \notin\{s(1, \delta), s(2, \delta), \ldots\} \\ f(n, \delta), & \text { if } k=s(n, \delta) .\end{cases}
$$

Now form $G=G(\mathbf{M})$, that is, the group generated by the $v(k, \delta)$ and the finitely nonzero sequences. To recapitulate, $G$ is a hyper-Archimedean $\ell$-group. 
Suppose that there is a hyper-Archimedean $\ell$-group $H$ with unit $u>0$ containing $G$ as an $\ell$-subgroup; (recall that the unit must be strong!) Then by our lemma we may assume that $H$ itself is an $\ell$-subgroup of $P$. Furthermore, we may, without loss of generality, assume that $u=(u(1), u(2), \ldots)$ where $1<u(1)<u(2)<\cdots$.

For each $\delta$ there is a positive integer $m(\delta)$ for which $m(\delta) u>v(\delta)$, so that $m(\delta) u(s(k, \delta)) \geq f(k, \delta)$, for each $k=1,2, \ldots$ Consider, in particular the case of $f(k, \delta)=u(t(k))^{2}$; (recall $t$, the bounding sequence!). Observe that

$$
m(\delta) u(s(k, \delta)) \geq u(t(k))^{2} \geq u(s(k, \delta))^{2},
$$

for each $k=1,2, \ldots$, since $u$ is increasing. But this implies that $m(\delta) \geq$ $u(s(k, \delta))$, for each $k$, which means that $u$ has a bounded subsequence; this is impossible.

Therefore no such adjunction of a unit is possible for $G=G(\mathbf{M})$.

The reader may now easily verify the following; $G$ is the example discussed above:

I. $G$ is not an $\ell$-subgroup of any Archimedean $\ell$-group with a strong unit.

II. $G$ cannot be realized as an $\ell$-subgroup of real-valued functions so that for each $0<g \in G$ the positive range values of $g$ are contained in an open interval $(a, b)$ with $a>0$. (If it could be so realized one could embed it in a hyper-Archimedean $\ell$-group with unit. Thus Conrad's open question 2) in $\left[\mathrm{C}_{3}\right]$ is settled.)

We say that a real-valued function $f$ on a set $I$ is a step function if its range is finite. Let $\mathbf{S}$ denote the class of ${ }^{\bullet} \ell$-groups which admit a representation as real-valued step functions on some set $I$.

III. $G \notin S$. (The group of all real-valued step functions defined on a set $I$ is hyper-Archimedean with unit. This answers open question 1) from $\left[\mathrm{C}_{3}\right]$.)

IV. $G$ cannot be represented as an $\ell$-group of bounded real-valued functions.

V. $G^{v}$ is a hyper-Archimedean vector lattice that does not belong to $S$. (See $[\mathrm{Ba}]$ and $[\mathrm{Be}]$ for other examples of such hyper-Archimedean vector lattices.)

VI. $G$ cannot be embedded in a hyper-Archimedean $f$-ring with no nonzero nilpotent elements. (Such an $f$-ring $R$ has a representation by real-valued functions so that every positive range of an element of $R$ is contained in an interval $(a, b)$ with $a>0$.)

A Specker $\ell$-subgroup $K$ of the hyper-Archimedean $\ell$-group $H$ is an $S$ kernel if $H$ is an $a$-extension of $K$; (meaning that for each $h>0$ in $H$ there is a $0<k \in K$ which generates the same convex $\ell$-subgroup as $h$.) It is shown in [CM] that $H$ admits an $S$-kernel precisely when it can be embedded as a convex $\ell$-subgroup of a hyper-Archimedean $\ell$-group with a unit. Thus:

VII. $G$ does not admit an $S$-kernel. 
Finally, we make three observations. Using a similar construction a student, David Nelson, has produced a Specker group $S$ with an $a$-subgroup $H$ in which no $a$-subgroup is Specker.

To underscore the significance of our example even further, we should point out that since $G=G(\mathbf{M})$ is a hyper-Archimedean subdirect product of integers it has a unique addition; that is to say, the natural group operation on $G$ is the only one for which the underlying lattice $(G, \vee, \wedge)$ is an $\ell$-group with identity 0 . (See $[C D]$.)

Elsewhere we will explore necessary and sufficient conditions for a hyperArchimedean $\ell$-group to admit an embedding as an $\ell$-subgroup of a hyperArchimedean $\ell$-group with unit.

\section{ACKNOWLEDGMENT}

This research was done while the second author was a Stouffer Visiting Professor at the University of Kansas during the academic year 1986-1987. He would like to thank his colleagues there for their warm reception.

\section{REFERENCES}

[AF] M. Anderson and T. Feil, Lattice-ordered groups, Reidel, Dordreacht, 1988.

[Ba] K. A. Baker, Topological methods in the algebraic theory of vector lattices, Thesis, Harvard University, 1966.

[Be] S. J. Bernau, On hyper-archimedean vector lattices, Proc. Kon. Nederl. Akad. v. Wetensch. 77 (1974), 40-43.

[BKW] A. Bigard, K. Keimel and S. Wolfenstein, Groupes et anneaux réticulés, Lecture Notes in Math., vol. 608, Springer, Berlin, 1977.

[B $\left.\mathrm{B}_{1}\right] \quad$ R. Bleier, Minimal vector lattices, Bull. Austral. Math. Soc. 5 (1971), 411-413.

$\left[\mathrm{C}_{1}\right] \quad$ P. Conrad, Some structure theorems for lattice-ordered groups, Trans. Amer. Math. Soc. 99 (1961), 212-240.

$\left[\mathrm{C}_{2}\right]$ - Minimal vector lattices, Bull. Austral. Math. Soc. 4 (1971), 35-39.

$\left[C_{3}\right]$ - Epi-archimedean groups, Czeachoslovak Math. J. 24 (99) (1974), 192-218.

[CD] P. Conrad and M. Darnel, $\ell$-groups with unique addition, Proc. First Symp. Ordered Algebraic Structures, Helderman, Berlin, 1986, pp. 15-27.

[CHH] P. Conrad, J. Harvey, and W. C. Holland, The Hahn embedding theorem for lattice-ordered groups, Trans. Amer. Math. Soc. 108 (1963), 143-169.

[CM] P. Conrad and J. Martinez, Signatures and discrete lattice-ordered groups, Alg. Universalis (to appear).

Department of Mathematics, University of Kansas, Lawrence, Kansas 66045

Department of Mathematics, University of Florida, Gainesville, Florida 32611 